CLINICAL STUDY

\title{
Improved glycemic control induced by both metformin and repaglinide is associated with a reduction in blood levels of 3-deoxyglucosone in nonobese patients with type 2 diabetes
}

Lian Engelen $^{1,2, *}$, Søren S Lund ${ }^{3, *}$, Isabel Ferreira ${ }^{1,2,4}$, Lise Tarnow ${ }^{3}$, Hans-Henrik Parving ${ }^{5,6}$, Jørgen Gram ${ }^{7,8}$, Kaj Winther ${ }^{9}$, Oluf Pedersen ${ }^{6,10,11}$, Tom Teerlink ${ }^{12}$, Rob Barto ${ }^{12}$, Coen D A Stehouwer ${ }^{1,2}$, Allan A Vaag ${ }^{3,13}$ and Casper G Schalkwijk ${ }^{1,2}$

${ }^{1}$ Cardiovascular Research Institute Maastricht (CARIM) ${ }^{2}$ Laboratory for Metabolism and Vascular Medicine, Department of Internal Medicine, Maastricht University Medical Centre, Debeyelaan 25, PO Box 5800, 6202AZ Maastricht, The Netherlands, ${ }^{3}$ Steno Diabetes Center, 2820 Gentofte, Denmark, ${ }^{4}$ Department of Clinical Epidemiology and Medical Technology Assessment (KEMTA), Maastricht University Medical Centre, 6202 Maastricht, The Netherlands, ${ }^{5}$ Department of Medical Endocrinology, University Hospital of Copenhagen, 2100 Copenhagen, Denmark, ${ }^{6}$ Faculty of Health Science, University of Aarhus, Aarhus, Denmark, ${ }^{7}$ Department of Thrombosis Research, University of Southern Denmark, Esbjerg, Denmark, ${ }^{8}$ Department of Clinical Biochemistry, Hospital of South West Denmark, 6700 Esbjerg, Denmark, ${ }^{9}$ Department of Clinical Biochemistry, Frederiksberg Hospital, 2000 Frederiksberg, Denmark, ${ }^{10}$ Hagedorn Research Institute, 2820 Gentofte, Denmark, ${ }^{11}$ Faculty of Health Sciences, Institute of Biomedical Science, University of Copenhagen, 1165 Copenhagen, Denmark, ${ }^{12}$ Department of Clinical Chemistry, VU University Medical Center, 1007 MB Amsterdam, The Netherlands and ${ }^{13}$ Department of Endocrinology, University of Lund, 20502 Malmö, Sweden

(Correspondence should be addressed to C G Schalkwijk at Laboratory for Metabolism and Vascular Medicine, Maastricht University Medical Centre; Email: c.schalkwijk@intmed.unimaas.nl)

*(L Engelen and S S Lund contributed equally to this work)

\begin{abstract}
Objective: Metformin has been reported to reduce $\alpha$-dicarbonyls, which are known to contribute to diabetic complications. It is unclear whether this is due to direct quenching of $\alpha$-dicarbonyls or to an improvement in glycemic control. We therefore compared the effects of metformin versus repaglinide, an antihyperglycemic agent with an insulin-secreting mechanism, on the levels of the $\alpha$-dicarbonyl 3-deoxyglucosone (3DG).

Methods: We conducted a single-center, double-masked, double-dummy, crossover study involving 96 nonobese patients with type 2 diabetes. After a 1-month run-in on diet-only treatment, patients were randomized to either repaglinide ( $6 \mathrm{mg}$ daily) followed by metformin ( $2 \mathrm{~g}$ daily) or vice versa each during 4 months with a 1-month washout between interventions.

Results: 3DG levels decreased after both metformin ( $-19.3 \%$ (95\% confidence interval (CI): -23.5 , $-14.8)$ ) and repaglinide $(-20.8 \%(95 \% \mathrm{CI}:-24.9,-16.3))$ treatments, but no difference was found between treatments $(1.8 \%(95 \% \mathrm{CI}:-3.8,7.8))$. Regardless of the treatment, changes in glycemic variables were associated with changes in 3DG. Specifically, 3DG decreased by $22.7 \%$ (95\% CI: 19.0 , $26.5)$ per s.D. decrease in fasting plasma glucose (PG), by $20.0 \%$ (95\% CI: 16.2, 23.9) per s.D. decrease in seven-point mean plasma glucose, by $22.5 \%$ (95\% CI: 18.6, 26.6) per s.D. decrease in area under the curve for PG, by $17.2 \%$ (95\% CI: 13.8, 20.6) per s.D. decrease in HbAlc, and by $10.9 \%$ (95\% CI: 6.4 , 15.5) per S.D. decrease in Amadori albumin. In addition, decreases in 3DG were associated with decreases in advanced glycation endproducts and endothelial markers.

Conclusion: Improved glycemic control induced by both metformin and repaglinide is associated with a reduction in 3DG levels in nonobese individuals with type 2 diabetes. This may constitute a shared metabolic pathway through which both treatments have a beneficial impact on the cardiovascular risk.
\end{abstract}

European Journal of Endocrinology 164 371-379

\section{Introduction}

The mechanisms underlying the development of vascular complications in diabetes are not completely understood. A potential mechanism by which hyperglycemia and its immediate biochemical sequelae induce vascular complications involves the increased formation of advanced glycation endproducts (AGEs) (1).
The deleterious effects of AGEs are attributed to direct modification of extracellular matrix proteins, resulting in collagen cross-links, which lead to increased vascular stiffness (1). In addition, binding of AGEs to a variety of cell-surface receptors, such as the receptor for AGEs, triggers signal transduction pathways and the production of reactive oxygen species, resulting in proinflammatory cellular responses (2). Given these 
deleterious effects of AGEs, several potential inhibitors (e.g. aminoguanidine, pyridoxamine, and cross-link breaker ALT-711) have been developed to reduce the clinical impact of AGEs (3).

Although the formation of AGEs is complex, it is well established that $\alpha$-dicarbonyl compounds such as 3-deoxyglucosone (3DG) and methylglyoxal are reactive intermediates in the formation of AGEs (4). The levels of 3DG have been shown to be elevated in patients with diabetes $(5,6)$, particularly in those with diabetic microangiopathy, such as retinopathy (5), neuropathy (5), and nephropathy $(5,7)$.

Aminoguanidine, which is a small hydrazine-like molecule, inhibits the formation of AGEs by interaction with and quenching of dicarbonyl compounds (8). Metformin, an antihyperglycemic agent, which enhances insulin sensitivity and lowers hepatic glucose output, may also have antiglycation properties, with a working mechanism similar to aminoguanidine due to a similarity in structure between both the agents (9-11). The observation of reduced levels of methylglyoxal in patients using metformin as compared with controls supports this view (9). Based on this additional effect, the beneficial impact of metformin on cardiovascular complications of type 2 diabetes, as for instance reported in the UKPDS study (12), could be, at least in part, ascribed to its specific effects on detoxifying dicarbonyl compounds, thereby preventing AGE accumulation. However, a reduction in dicarbonyls following treatment with metformin may also derive from an improvement in glycemic control.

In view of these considerations, and in order to disentangle those two possible operating mechanisms, we have investigated, in a single-center, double-masked, double-dummy, crossover study in nonobese patients with type 2 diabetes (initially investigating the hypothesis of equivalent antihyperglycemic effects of metformin and repaglinide; ClinicalTrials.gov Identifier: NCT00118950) (13-15)) i) the effects of metformin versus repaglinide - an antihyperglycemic agent with a different working mechanism (i.e. stimulating insulin secretion) - on the levels of 3DG; ii) the associations of treatment-induced changes in glycemic variables (i.e. fasting plasma glucose (PG), seven-point mean PG, area under the curve for PG (AUC-PG), HbAlc, and Amadori albumin) with changes in 3DG levels; and iii) the associations between changes in 3DG levels and changes in biomarkers of advanced glycation, endothelial dysfunction, and low-grade inflammation.

\section{Materials and methods}

\section{Study design}

In 2001-2003, we conducted a single-center, randomized, double-masked, double-dummy, crossover study with 96 nonobese (body mass index $\leq 27 \mathrm{~kg} / \mathrm{m}^{2}$ ) insulin-naive individuals with type 2 diabetes as described in detail elsewhere (13-15). Briefly, we included 96 subjects who had type 2 diabetes diagnosed when 40 years of age or older, had no history of ketonuria, or diabetic ketoacidosis and, at enrollment, presented with a fasting serum C-peptide $\geq 300 \mathrm{pmol} / \mathrm{l}$ or a nonfasting or glucagon-stimulated C-peptide $\geq 600 \mathrm{pmol} / \mathrm{l}$. After enrollment, patients stopped intake of oral hypoglycemic agents and underwent a 1-month run-in period on diet-only treatment. After the run-in period, patients were randomized to receive either $2 \mathrm{mg}$ repaglinide three times daily plus placebo metformin followed by $1 \mathrm{~g}$ metformin twice daily plus placebo repaglinide, or vice versa, each treatment for a period of 4 months with a 1-month washout period between interventions.

Out of the 96 included patients, 93 and 89 patients initiated a treatment period with metformin and repaglinide respectively. Throughout the course of treatments, 20 patients $(21 \%)$ dropped out, leaving 76 patients $(79 \%)$ who completed the whole trial; 83 and 82 patients completed a treatment period with metformin or repaglinide respectively. Ten (11\%) patients dropped out during metformin treatment, seven $(8 \%)$ during repaglinide treatment, and three (3\%) during the washout period; one after repaglinide and two after metformin treatment (Fig. 1) (14).

Results regarding the effects of treatments on trial's primary endpoint, HbAlc, and secondary endpoints, such as postprandial glucose and lipid metabolism, as well as markers of inflammation and endothelial dysfunction have been reported previously (13-15). This study focuses specifically on the effects of treatments on blood levels of 3DG, which due to the complexity of its assessment only became available recently. Measures to ensure proper blood sampling to enable future assessment of 3DG levels were planned prior to and undertaken during the trial (see also later).

The study was conducted in accordance with the Declaration of Helsinki and was approved by the ethics committee of Copenhagen County, Denmark.

\section{Blood sampling}

Patients were instructed to obtain seven-point homemonitored PG measurements (preprandial, $90 \mathrm{~min}$ postprandial, and prebedtime) 2 days per week during the treatment periods, which were used to calculate the mean PG levels (seven-point MPG).

Patients were further investigated at the Steno Diabetes Center (Gentofte, Denmark) where blood samples were taken during the last week before the patient entered a treatment period and on the last day of each treatment period. Blood samples were drawn with minimal venous occlusion between 0800 and $0900 \mathrm{~h}$ after a $10 \mathrm{~h}$ overnight fast and $25 \mathrm{~min}$ of supine rest. Additionally, during a standardized 0-6 h meal test, blood was withdrawn at $t=0,90,180$, 270 , and $360 \mathrm{~min}$, and used to calculate the AUC-PG 


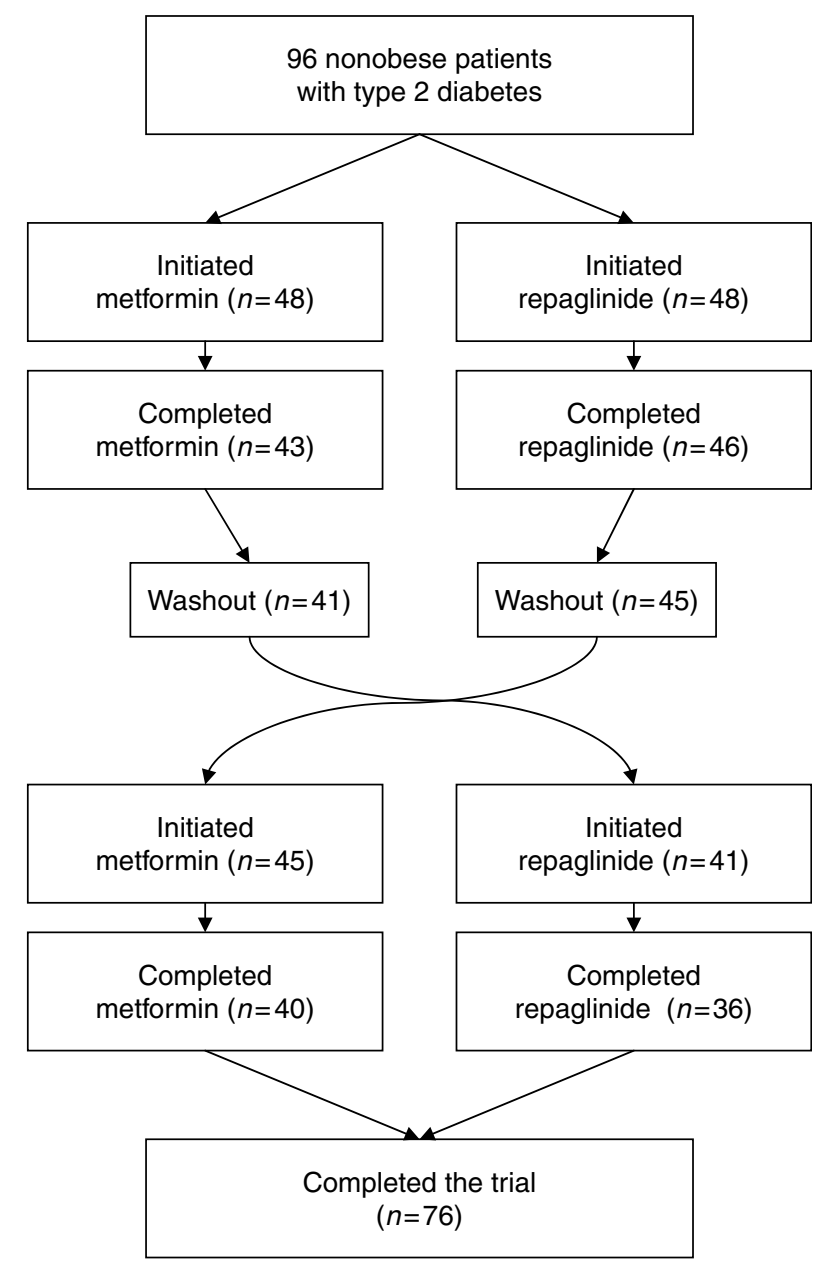

Figure 1 Study design and flowchart of the 96 randomized patients.

by the trapezoidal rule (i.e. $1.5 \mathrm{~h} \times(0.5 \times$ concentration $_{t=0 \mathrm{~h}}+$ concentration $_{t=1.5 \mathrm{~h}}+$ concentration $_{t=}$ $3.0 \mathrm{~h}+$ concentration $_{t=4.5 \mathrm{~h}}+0.5 \times$ concentration $_{t=}$ $6.0 \mathrm{~h}$ ) in $\mathrm{mmol} / \mathrm{l} \times \mathrm{h})(13)$.

Sodium citrate and EDTA plasma and serum samples were prepared by centrifugation at $2000 \boldsymbol{g}$ for $10 \mathrm{~min}$ at $20{ }^{\circ} \mathrm{C}$ and immediately frozen hereafter. Samples were stored at $-80{ }^{\circ} \mathrm{C}$ until analyses.

$\alpha$-dicarbonyls (such as $3 \mathrm{DG}$ ) react rapidly with arginine and lysine residues in proteins. Therefore, as the measurement of 3DG was anticipated prior to initiating the trial, $0.5 \mathrm{ml}$ whole blood was collected in specific blood collection tubes to be immediately mixed with $1.0 \mathrm{ml}$ of $1.2 \mathrm{~mol} / \mathrm{l}$ perchloric acid to precipitate the protein fraction and stored at $-80^{\circ} \mathrm{C}$ until assayed.

\section{Biochemical analyses}

3DG was measured using liquid chromatographytandem mass spectrometry (LC-tandem MS) (6). After thawing the acidified blood samples, the samples were centrifuged $(5 \mathrm{~min}$ at $20000 \boldsymbol{g})$ and $80 \mu \mathrm{l}$ of the supernatant was mixed with $100 \mu \mathrm{l}$ of the internal standard solution $(1 \mu \mathrm{M} / \mathrm{l}$ 2,3-pentanedione dissolved in ethanol). After the addition of $20 \mu \mathrm{l}$ of the derivatization reagent $(10 \mathrm{mM} / \mathrm{l}$ 2,4-dinitrophenylhydrazine dissolved in $1.2 \mathrm{M} / \mathrm{l}$ perchloric acid), the samples were incubated for $16 \mathrm{~h}$ at room temperature. Chromatographic separation was performed on an Xterra MS C1 8 column $(4.6 \times 50 \mathrm{~mm}, 3.5 \mu \mathrm{m}$ particle size) (Waters, Milford, MA, USA) using a linear gradient from 40 to $95 \%$ acetonitrile in water over 9 min at a flow rate of $1 \mathrm{ml} / \mathrm{min}$. Mass transitions of 521.1-431.0 and 521.1-182.1 for 3DG and 459.1-182.1 for the internal standard were monitored in negative ion mode. The derivatization and the chromatographic separation were performed at a low $\mathrm{pH}$, minimizing the chance of 3DG formation from sugars and fructosyl-lysine at this stage (4). To test the recovery of 3DG, calibration curves prepared in whole blood (two different samples) were compared with a calibration curve prepared in water. The slopes of the calibration curves in whole blood were not significantly different from the slope of the calibration curve in water. As part of validation of the method, we performed a comparison with a previously published method, based on derivatization with 1,2-diamino-4,5-dimethoxybenzene (DDB) followed by HPLC analysis with fluorescence detection (16). In a comparison of perchloric acid supernatants of 44 whole blood samples, results obtained with both methods were highly correlated $(r=0.92 ; P<0.001)$. Comparison of both the methods by the Deming regression showed that intercept and slope of the regression line were not significantly different from 0 and 1 respectively. Mean \pm S.D. 3DG concentrations obtained by LC-MS/MS and LC-fluorescence were $188 \pm 72$ and $181 \pm 61 \mathrm{nM}$ respectively. Values obtained by both the methods were not significantly different. Intra- and inter-assay coefficients of variation were $<10 \%$.

PG (at study visits: venous samples; home-monitored: fresh capillary blood samples) was measured with the glucose-oxidase biosensor method (EuroFlash handdevice, LifeScan, Milpitas, CA, USA; calibrated for PG with the YSI Model 2300 Glucose Analyser, YSI, Inc., Yellow Springs, OH, USA). The coefficient of variation between test strips was $<10 \%$. HbAlc was measured by ion-exchange HPLC method traceable to the Diabetes Control and Complication Trial (DCCT) standard (BioRad VARIANT method, Bio-Rad Diagnostics Group). Amadori albumin was measured in sodium citrate plasma with ELISA (17).

$N^{\varepsilon}$-(carboxymethyl)lysine (CML) and $N^{\varepsilon}$-(carboxyethyl)lysine (CEL) residues in plasma proteins were measured by stable isotope dilution tandem MS (18).

The concentration of pyrraline in the protein-free plasma fraction was determined by UPLC-tandem MS. Because a stable isotope pyrraline internal standard was not commercially available, we have chosen, after extensive validation, $\left[{ }^{13} \mathrm{C}_{9}\right]-3$-nitrotyrosine as a nonanalog internal standard. A $25 \mu$ plasma fraction 
was mixed thoroughly with $12.5 \mu \mathrm{l}$ internal standard (3.9 $\mu \mathrm{M}\left[{ }^{13} \mathrm{C}_{9}\right]$-3-nitrotyrosine) and $500 \mu \mathrm{l}$ methanol: acetonitrile (1:3, by volume). Proteins were removed by centrifugation $(20 \mathrm{~min}$ at $14000 \boldsymbol{g})$ and the supernatant was evaporated to dryness at $65^{\circ} \mathrm{C}$ under a constant stream of nitrogen. The residue was dissolved in $150 \mu \mathrm{l}$ water. Reversed-phase chromatography was carried out using an Acquity UPLC $\mathrm{BEH} \mathrm{C}_{18}$ column $(2.1 \times 50 \mathrm{~mm}, 1.7 \mu \mathrm{m}$ particle size, Waters $)$ with $10 \mathrm{mM}$ formic acid as solvent $\mathrm{A}$ and acetonitrile as solvent B. A linear gradient was started at $99 \%$ solvent A, which was changed in $1.5 \mathrm{~min}$ to $95.5 \%$ solvent A. After cleaning the column with $45 \%$ solvent B during 4 min the column was equilibrated for $2 \mathrm{~min}$ at the initial conditions. Injection volume was $2 \mu \mathrm{l}$ and column temperature was set at $48{ }^{\circ} \mathrm{C}$. Detection was carried out using a Xevo TQ tandem mass spectrometer (Waters) in electrospray-positive mode. Multiple reaction monitoring transitions were set at 236.10-189.15 for $\left[{ }^{13} \mathrm{C}_{9}\right]$-3-nitrotyrosine and at $255.10-175.20$ for pyrraline. Capillary voltage was set at $0.5 \mathrm{kV}$ and a cone voltage of 14 and $10 \mathrm{~V}$ was used for $\left[{ }^{13} \mathrm{C}_{9}\right]-3-$ nitrotyrosine and pyrraline respectively. We tested recovery (and thus matrix effects), based on additions of pyrraline standard to different matrices. Mean recovery, tested in three different matrices, was 98.5 $\pm 5.3 \%$. Linearity was tested in 11 different matrices by adding known pyrraline concentrations between 0 and $300 \mathrm{nM}$. Mean response factor was $1.44 \pm 0.11$ and $1.33 \pm 0.06$ in plasma and water respectively. The intraassay variation was $3.5 \%$ as determined in a plasma sample $(n=10)$ with a mean pyrraline concentration of $89.8 \mathrm{nM}$. The inter-assay variation was $4.9 \%$ as determined in a plasma sample $(n=7)$ with a mean pyrraline concentration of $85.2 \mathrm{nM}$. The lower limit of quantification was $19.5 \mathrm{fmol}$ at an injection volume of $5 \mu \mathrm{l}($ signal to noise ratio $(\mathrm{s} / \mathrm{N})=6)$.

For the evaluation of endothelial dysfunction, we measured plasma levels of plasminogen activator inhibitor-1 (PAI1 or SERPINE) antigen (PAI1-ag), tissuetype plasminogen activator antigen (tPA-ag; TintElize PAI-1 and Immulyse tPA respectively; Biopool, Umeå, Sweden), and von Willebrand factor (vWf) antigen using a highly sensitive immunoassay (19). Serum levels of soluble intercellular adhesion molecule-1 (sICAM1), soluble vascular cell adhesion molecule-1 (sVCAM1), and soluble E-selectin (selectin E; sSELE) were measured using commercially available ELISA kits (Diaclone, Besançon, France).

For the evaluation of low-grade inflammation, we measured the levels of serum C-reactive protein (CRP) using a highly sensitive enzyme immunoassay (19). Plasma levels of tumor necrosis factor- $\alpha$ (TNF- $\alpha$ ) and serum levels of interleukin 6 (IL6) were measured using commercially available ELISA kits (Quantikine High Sensitivity; R\&D Systems, Oxon, UK) and fibrinogen was measured using the MultifibrenU reagent (Dade Behring, Marburg, Germany).

\section{Statistical analysis}

Outcome variables were evaluated after the run-in period (i.e. before initiating treatment in the first period), referred to as first-period baseline; after the washout period (i.e. before initiating treatment in the second period), referred to as second-period baseline; and on the last day of each treatment period. Treatment effects were estimated as change from first-period baseline.

Comparisons between first- and second-period baseline levels of the metabolic variables examined in this study were performed with the use of Student's $t$-test. We used a linear mixed model, with subject as a random effect, to compare the treatment effects of metformin versus repaglinide on changes in 3DG levels. Treatment type (metformin or repaglinide), treatment sequence (metformin followed by repaglinide or vice versa), the period effect, and the first-period baseline levels of 3DG were included as fixed effects. The extent to which the potential occurrence of unexpected carryover effects might have influenced sequence and period effects was thereby taken into account. Furthermore, analyses with a linear mixed model enabled information from incomplete blocks (i.e. those with dropouts) to be included (i.e. both within- and between-subject information was included).

Associations of changes in other metabolic variables with changes in 3DG were investigated by adding baseline and changes in glycemic variables (i.e. fasting (FPG), seven-point mean (MPG), AUC-PG, HbAlc, and Amadori albumin) to our initial model. Associations between changes in 3DG and changes in biomarkers of advanced glycation (i.e. CML, CEL, and pyrraline), endothelial dysfunction (i.e. PAI1-ag, tPA-ag, vWf, sICAM1, sVCAM1, and sSELE), and low-grade inflammation (i.e. CRP, TNF- $\alpha$, IL6, and fibrinogen) were evaluated in a similar way. Thereby, associations of changes in these variables with changes in 3DG levels were thus adjusted for treatment type and sequence, the period effect, and first-period baseline levels of both 3DG and the other studied metabolic variable. In order to enable comparison of the strength of these associations, we used standardized (i.e. (subjects' value - population mean)/s.D.) values of changes in the metabolic variables.

Variables with skewed distribution (i.e. 3DG, pyrraline, PAI1-ag, sICAM1, sVCAM1, sSELE, CRP, TNF- $\alpha$, and IL6) were logarithmically transformed prior to analyses and are presented as median and interquartile range (IQR). Treatment effects on 3DG and associations between changes in other metabolic variables and changes in 3DG and between changes in 3DG and changes in biomarkers with skewed distribution are presented as percentages due to required back transformation of the regression coefficients.

All statistical analyses were carried out using the Statistical Package for Social Sciences, version 15.0 (SPSS, Inc., Chicago, IL, USA). 


\section{Results}

Patients' characteristics at enrollment are shown in Table 1. A more detailed description of patients' characteristics (e.g. use of medication) at enrollment as well as of adverse events during the trial have been presented elsewhere (14).

Table 2 shows first- (i.e. after 1-month run-in on diet-only treatment) and second-period (i.e. after 1-month washout on diet-only treatment) baseline data of 3DG and the other metabolic variables examined, according to each treatment sequence. No significant differences were found between first- and second-period baseline data.

\section{Effects of metformin or repaglinide treatment on $3 D G$}

3DG levels decreased after both metformin $(-19.3 \%$ (95\% confidence interval (CI): $-23.5,-14.8)$ ) and repaglinide $(-20.8 \% \quad(95 \%$ CI: $-24.9,-16.3))$ treatments (Fig. 2), but no significant difference was found between the two treatments $(1.8 \% \quad(95 \%$ CI: $-3.8,7.8)$ ).

Table 1 Clinical characteristics of the study participants at enrollment. Data are expressed as mean \pm s.D., median (range) or percentages. Data are stratified according to the treatment sequence: repaglinide followed by metformin or vice versa.

\begin{tabular}{|c|c|c|}
\hline & $\begin{array}{l}\text { Repaglinide } \\
\text { followed by } \\
\text { metformin } \\
\quad(n=48)\end{array}$ & $\begin{array}{l}\text { Metformin } \\
\text { followed by } \\
\text { repaglinide } \\
(n=48)\end{array}$ \\
\hline Men/women $(n)$ & $36 / 12$ & $37 / 11$ \\
\hline Age (years) & $63.3 \pm 8.9$ & $59.5 \pm 9.3$ \\
\hline $\begin{array}{l}\text { Known duration of } \\
\text { diabetes (years) }\end{array}$ & $5(0-28)$ & $3(0-22)$ \\
\hline Body mass index $\left(\mathrm{kg} / \mathrm{m}^{2}\right)$ & $24.8 \pm 1.9$ & $24.7 \pm 2.1$ \\
\hline HbAlc (\%) & $7.6 \pm 0.9$ & $7.3 \pm 0.8$ \\
\hline Smoking, $n(\%)$ & $28(58)$ & $27(56)$ \\
\hline \multicolumn{3}{|l|}{ Late diabetic complications (\%) } \\
\hline $\begin{array}{l}\text { Retinopathy (none/simplex/ } \\
\text { proliferative) }\end{array}$ & $65 / 33 / 2$ & $73 / 21 / 6$ \\
\hline Macroangiopathy & 21 & 23 \\
\hline $\begin{array}{l}\text { Nephropathy (normo-/micro-/ } \\
\text { macroalbuminuria) }\end{array}$ & $71 / 25 / 4$ & $73 / 23 / 4$ \\
\hline Neuropathy & 75 & 71 \\
\hline \multicolumn{3}{|c|}{ Prestudy anti-hyperglycaemic treatment (\%) } \\
\hline Diet-only & 19 & 15 \\
\hline Metformin & 13 & 8 \\
\hline Repaglinide & 8 & 0 \\
\hline Sulfonylurea & 44 & 63 \\
\hline Metformin plus sulfonylurea & 17 & 15 \\
\hline \multicolumn{3}{|c|}{ Ongoing nonstudy medication (\%) } \\
\hline Antihypertensive medication & 63 & 46 \\
\hline Lipid-lowering medication & 35 & 25 \\
\hline Aspirin & 44 & 46 \\
\hline
\end{tabular}

${ }^{a}$ Note that the proportion of patients with diabetic complications have been changed $(13,15)$ from the original publication (14) after checking clinical signs with patients' medical records.

\section{Associations between changes in glycemic variables and changes in $3 D G$}

To explore a potential pathway through which both treatments could have induced decreases in 3DG levels, we investigated the associations between changes in a range of glycemic variables and changes in 3DG.

Changes in all glycemic variables were positively associated with changes in 3DG (Table 3). Specifically, for each S.D. decrease in FPG, 3DG decreased by $22.7 \%$ (95\% CI: 19.0, 26.5). Similarly, 3DG decreased by $20.0 \%$ (95\% CI: 16.2, 23.9) per S.D. decrease in sevenpoint MPG, by $22.5 \%(95 \%$ CI: $18.6,26.6)$ per S.D. decrease in AUC-PG, and by $17.2 \%$ (95\% CI: 13.8, 20.6) per S.D. decrease in HbAlc. Noticeably, the magnitudes of these associations were similar. In addition, for each s.D. decrease in Amadori albumin, 3DG decreased by $10.9 \%$ (95\% CI: 6.4, 15.5).

\section{Associations between changes in 3DG and changes in biomarkers of advanced glycation, endothelial dysfunction, and inflammation}

To explore potential pathways through which decreases in 3DG levels could influence the cardiovascular risk profile, we investigated the associations between changes in 3DG and changes in biomarkers of advanced glycation, endothelial dysfunction, and inflammation.

For each s.D. decrease in 3DG, CML decreased by 0.17 S.D. $(95 \%$ CI: $0.01,0.33)$, but no associations were found between changes in 3DG and changes in CEL $(0.08$ s.D. $(95 \%$ CI: $-0.07,0.24))$ or pyrraline $(-1.8 \%$ (95\% CI: - 11.4, 8.8)) (Table 4).

Furthermore, the treatment-induced reduction in 3DG was associated with a reduction in the endothelial markers PAI-1-ag, tPA-ag, sVCAM-1, sSELE, but not with changes in $v W f$ and sICAM-1. In addition, we found that treatment-induced reduction in 3DG was not associated with statistically significant reductions in the inflammatory markers TNF- $\alpha$, CRP, IL6, and fibrinogen (Table 5).

\section{Additional analyses}

Additional adjustment for changes in body mass, which differed between treatments (14) and may affect the metabolic variables examined herein, did not substantially influence our results. Specifically, after the additional adjustment for changes in body mass, 3DG levels decreased after both metformin $(-18.0 \%(95 \% \mathrm{CI}:-22.4,-13.6))$ and repaglinide $(-22.0 \%$ (95\% CI: $-26.1,-17.7))$ treatments, but no significant difference was found between the two treatments $(-4.8 \%$ (95\% CI: -10.4 , 1.1)). In addition, adjustment for changes in body mass did not substantially influence the associations between changes in glycemic variables and biomarkers of advanced glycation, endothelial dysfunction, and lowgrade inflammation and changes in 3DG (data not shown). 
Table 2 First- and second-period baseline values of the studied metabolic variables. Data are expressed as mean \pm S.D. or median (interquartile range), and stratified according to the treatment sequence: repaglinide followed by metformin (RE-MET) or vice versa (MET-RE).

\begin{tabular}{|c|c|c|c|c|c|c|}
\hline & \multicolumn{2}{|c|}{ First-period baseline } & \multicolumn{2}{|c|}{ Second-period baseline } & \multicolumn{2}{|c|}{$P$ value } \\
\hline & RE-MET $(n=48)$ & MET-RE $(n=48)$ & RE-MET $(n=45)$ & MET-RE $(n=41)$ & RE-MET & MET-RE \\
\hline 3-Deoxyglucosone (nM) & $1164(945 ; 1574)$ & $1185(865 ; 1487)$ & $1122(854 ; 1648)$ & $1089(903 ; 1403)$ & 0.855 & 0.539 \\
\hline $\mathrm{FPG}(\mathrm{mmol} / \mathrm{l})$ & $14.0 \pm 4.0$ & $12.8 \pm 3.5$ & $14.4 \pm 4.4$ & $13.6 \pm 4.2$ & 0.344 & 0.074 \\
\hline Seven-point MPG $(\mathrm{mmol} / \mathrm{l})^{\mathrm{a}}$ & $13.0 \pm 4.4$ & $12.2 \pm 3.1$ & $13.6 \pm 4.0$ & $11.5 \pm 3.2$ & 0.208 & 0.121 \\
\hline AUC-PG $\left(\text { time }^{\mathrm{a}} \mathrm{mmol} / \mathrm{l}\right)^{\mathrm{b}}$ & $6008 \pm 1652$ & $5498 \pm 1528$ & $6083 \pm 1760$ & $5630 \pm 1658$ & 0.692 & 0.438 \\
\hline HbAlc (\%) & $8.2 \pm 1.2$ & $7.8 \pm 0.8$ & $8.0 \pm 1.5$ & $7.8 \pm 1.2$ & 0.246 & 0.656 \\
\hline Amadori albumin $(\mathrm{U} / \mathrm{ml})$ & $50 . \overline{2} \pm 11.1$ & $48 . \overline{7} \pm 12.2$ & $48.6 \pm 12.4$ & $48 . \overline{9} \pm 10.9$ & 0.346 & 0.873 \\
\hline CML (mmol/mol lysine) & $0.069 \pm 0.015$ & $0.068 \pm 0.015$ & $0.070 \pm 0.012$ & $0.070 \pm 0.015$ & 0.515 & 0.064 \\
\hline CEL (mmol/mol lysine) & $0.016 \pm 0.003$ & $0.016 \pm 0.003$ & $0.016 \pm 0.003$ & $0.016 \pm 0.003$ & 0.713 & 0.845 \\
\hline Pyrraline (nM) & $17.4(9.6 ; 26.3)$ & $17.6(11.6 ; 26.0)$ & $20.3(13.0 ; 27.1)$ & $17.4(10.6 ; 25.6)$ & 0.104 & 0.993 \\
\hline
\end{tabular}

AUC, area under the curve during the $6 \mathrm{~h}$ meal test; $\mathrm{CML}, N^{\varepsilon}$-(carboxymethyl)lysine; CEL, $N^{\varepsilon}$-(carboxyethyl)lysine.

an the RE-MET group, $n=36$ and $n=32$ and in the MET-RE group $n=37$ and $n=24$ for the first- and second-period baseline respectively.

${ }^{\mathrm{b}}$ In the RE-MET group, $n=46$ and $n=42$ and in the MET-RE group $n=45$ and $n=38$ for the first- and second-period baseline respectively.

\section{Discussion}

The present single-center, randomized, double-masked, double-dummy, crossover study of 96 nonobese insulinnaive individuals with type 2 diabetes comparing the effects of metformin and repaglinide treatment on blood levels of 3DG had three main findings. First, 3DG levels decreased to the same extent either after treatment with metformin or with repaglinide. Secondly, changes in several glycemic variables, i.e. FPG, seven-point MPG, AUC-PG, HbAlc, and Amadori albumin, were strongly and positively associated with these changes in 3DG levels. Thirdly, metformin- and repaglinide-induced changes in 3DG were positively associated with changes in CML (i.e. an AGE that can be formed by 3DG) and several markers of endothelial dysfunction (i.e. PAI1, tPA-ag, sVCAM1, and sSELE).

Given the similarities in structure between metformin and aminoguanidine, it has been proposed that metformin reacts with the dicarbonyl groups of
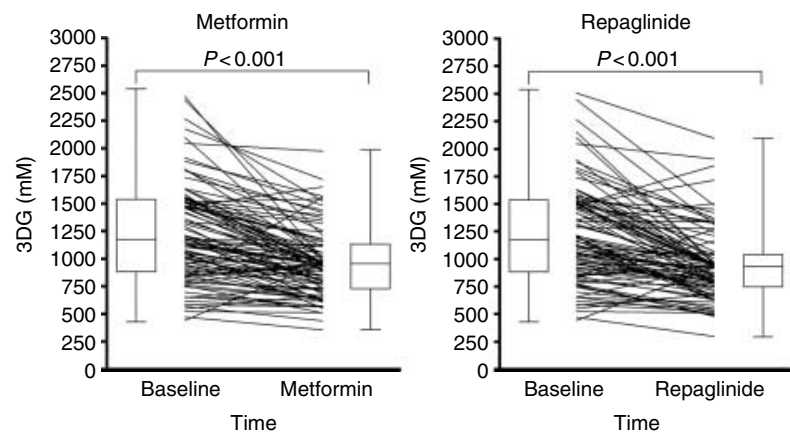

Figure 2 Levels of 3DG at baseline and after treatment with metformin or repaglinide. Boxes indicate the median values and the interquartile ranges and the whiskers represent the minimum and maximum values. $P$ values for differences between baseline and after 4 months of treatment were calculated using a linear mixed model (see 'Materials and methods' section). $\alpha$-oxoaldehydes in a similar way to aminoguanidine, thereby preventing the toxicity of these $\alpha$-oxoaldehydes and the subsequent production of AGEs (9-11). In this study, we indeed found a decrease in 3DG levels after treatment with metformin, which is in agreement with earlier observations of lower levels of $\alpha$-oxoaldehydes and AGEs in patients with type 2 diabetes treated with this drug $(9,11)$. Remarkably, we found a similar decrease in 3DG levels after treatment with another antihyperglycemic agent, repaglinide, which is a short-acting insulin secretagogue belonging to the meglitinide analogues.

The different working mechanisms of the antihyperglycemic effects of metformin and repaglinide suggest that improvement in glycemic control, rather than a specific effect of metformin on quenching of 3DG, results in a decrease in 3DG. This is concordant with a similar HbAlc-lowering effect of either treatment as previously reported (14) and further supported by our current findings, showing that changes in 3DG were strongly associated with changes in several glycemic variables, independently of treatment type, treatment sequence, and period effect.

Both metformin $(12,19)$ and repaglinide $(20,21)$ have been described to hold cardiovascular potentials in patients with type 2 diabetes, though a greater beneficial role has been attributed to metformin, which has also been previously shown in this study (15). Our current findings showing a similar beneficial effect of both drugs on 3DG levels, which are associated with diabetic microangiopathy (5-7), suggest that the metformin- or repaglinide-induced reduction in 3DG may explain part of the beneficial effect of both drugs on the cardiovascular risk profile.

We have also found a strong association between changes in AUC-PG and changes in 3DG. Postprandial rise in 3DG (6) and the association between postprandial glucose levels and levels of $\alpha$-oxoaldehydes including 3DG have been described previously (22). 
Table 3 Associations of changes in glycemic variables with percent changes in 3DG. The regression coefficient $\beta$ represents the percent change (i.e. decrease) in 3DG levels with a change (i.e. decrease) of 1 S.D. in the studied glycemic variables. All analyses were adjusted for treatment type, treatment sequence, the period effect, and baseline levels of 3DG and the studied glycemic variables.

\begin{tabular}{lccc}
\hline & \multicolumn{4}{c}{$\Delta$ 3DG } \\
\cline { 2 - 4 } & $\beta$ & $95 \% \mathrm{Cl}$ & $P$ value \\
\hline$\Delta$ FPG & 22.7 & $19.0 ; 26.5$ & $<0.001$ \\
$\Delta$ Seven-point MPG & 20.0 & $16.2 ; 23.9$ & $<0.001$ \\
$\Delta$ AUC-PG & 22.5 & $18.6 ; 26.6$ & $<0.001$ \\
$\Delta$ HbAlc & 17.2 & $13.8 ; 20.6$ & $<0.001$ \\
$\Delta$ Amadori albumin & 10.9 & $6.4 ; 15.5$ & $<0.001$ \\
\hline Cl, confidence interval; AUC, area under the curve during 6 h meal test.
\end{tabular}

In this regard it is important to emphasize that postprandial fluctuations in glucose levels have been thought to be more strongly associated with complications than high-glucose levels in general $(23,24)$. Although the exact mechanisms explaining the increased risk for cardiovascular disease due to glucose fluctuations are unknown, increased oxidative stress by glucose fluctuations during postprandial periods seems to be involved $(23,24)$. Such increases in oxidative stress may be due to increased formation of $\alpha$-oxoaldehydes (6). Therefore, both an increase in oxidative stress and dicarbonyl stress and consequent increased formation of AGEs may affect the vasculature and thus explain the increased cardiovascular risk related to postprandial fluctuations in glucose levels.

Two major pathways for the formation of 3DG have been described (25). 3DG can be formed in the polyol pathway, in which 3DG is a hydrolysis product of fructose-3-phosphate, and through the Maillard reaction. In the latter, the nonenzymatic glycation of protein amino groups leads to the formation of an Amadori product, which can then undergo multiple dehydration reactions and rearrangements to produce highly reactive carbonyl compounds such as 3DG. In accordance, we found that levels of Amadori albumin decreased after antihyperglycemic treatment and that this change was associated with a decrease in blood levels of 3DG. 3DG can react with free amino groups to form AGEs including CML and pyrraline (25). Indeed, our current findings show an association between changes in 3DG and changes in CML after glucoselowering treatment. However, we could not find such an association with changes in pyrraline. These differences might be explained by the fact that CML, rather than pyrraline, is a major AGE structure in 3DG-modified proteins (26).

Endothelial dysfunction and low-grade inflammation have been described as potential pathways to explain the increased risk for cardiovascular disease in both type $1(27,28)$ and type 2 diabetes $(29)$ and the increased formation of AGEs could have an important role in these pathways $(30,31)$. Indeed, our current results show an association between a glucose-lowering treatmentinduced reduction in 3DG and a reduction in markers of endothelial dysfunction - but not of low-grade inflammation - which further supports the view that this could constitute a pathway through which inhibition of the glycation pathway may have a beneficial impact on the cardiovascular risk profile. Moreover, from this study, we previously have shown that metformin had a more pronounced effect to lower such markers of endothelial dysfunction than did repaglinide despite overall similar glycemic control (i.e. independent of glycemic control) (15). Thus, together with our present data indicating equal potency of metformin and repaglinide to lower 3DG as well as an association between 3DG and markers of endothelial dysfunction, this suggests that metformin might have a potential beneficial influence on such markers of cardiovascular risk through both shared (glycemic) and specific (nonglycemic) pathways.

Some limitations to this study need to be mentioned. First, the lack of a 'nontreatment' or 'diet-only' arm does not enable us a full understanding of the importance of magnitude of the effects observed on 3DG levels after treatment with both metformin and repaglinide. Second, the study was not designed to assess the exact mediating effects of treatment-derived changes in the different glycemic variables on changes in 3DG. However, the strong and consistent associations between changes in the different variables - regardless of treatment type - and the fact that treatment has a similar effect on both 3DG and other glycemic variables suggest that changes in other glycemic variables, such as PG levels and HbAlc, at least partially explained the reduction in 3DG induced by metformin and repaglinide. Third, the presence of carryover effects in a trial with a crossover design could constitute such another limitation. However, the two treatment sequence groups (i.e. metformin followed by repaglinide or vice versa) were well balanced and comparable at each baseline measurement, and a possible carryover effect was adjusted for to the extent it influenced the effect of

Table 4 Associations of changes in 3DG with changes in biomarkers of advanced glycation. The regression coefficient $\beta$ represents the changes (in s.D. for CML and CEL; in \% for pyrraline) in the studied biomarker of advanced glycation with a change of 1 S.D. in 3DG levels. All analyses were adjusted for treatment type, treatment sequence, the period effect, and baseline levels of $3 D G$ and the studied biomarker of advanced glycation.

\begin{tabular}{lccc}
\hline & \multicolumn{3}{c}{$\Delta$ 3DG } \\
\cline { 2 - 4 } & $\beta$ & \multicolumn{1}{c}{$95 \% \mathrm{Cl}$} & $P$ value \\
\hline$\Delta N^{\varepsilon}-\mathrm{CML}$ & 0.17 & $0.01 ; 0.33$ & 0.044 \\
$\Delta N^{\varepsilon}-\mathrm{CEL}$ & 0.08 & $-0.07 ; 0.24$ & 0.286 \\
$\Delta$ Pyrraline & -1.8 & $-11.4 ; 8.8$ & 0.724 \\
\hline
\end{tabular}

$\mathrm{Cl}$, confidence interval. 
Table 5 Associations of changes in 3DG with changes in biomarkers of endothelial dysfunction and low-grade inflammation. The regression coefficient $\beta$ represents the changes (in S.D. for tPAantigen, vWf, fibrinogen; in \% for PAl1, sICAM, sVCAM, SSELE, CRP, TNF- $\alpha$, IL6) in the studied biomarker of endothelial dysfunction or low-grade inflammation with a change of 1 S.D. in 3DG levels. All analyses were adjusted for treatment type, treatment sequence, the period effect, and baseline levels of 3DG and the studied biomarker of endothelial dysfunction or low-grade inflammation.

\begin{tabular}{lccc}
\hline & \multicolumn{3}{c}{$\Delta$ 3DG } \\
\cline { 2 - 4 } & $\beta$ & $95 \%$ Cl & $P$ value \\
\hline Endothelial dysfunction & & & \\
$\Delta$ PAl1 & 19.1 & $1.3 ; 40.0$ & 0.034 \\
$\Delta$ tPA-ag & 0.17 & $0.02 ; 0.33$ & 0.029 \\
$\Delta$ vWf & 0.08 & $-0.10 ; 0.25$ & 0.383 \\
$\Delta$ sICAM1 & 3.9 & $-12.3 ; 23.0$ & 0.658 \\
$\Delta$ sVCAM1 & 20.5 & $1.5 ; 43.1$ & 0.034 \\
$\Delta$ sSELE & 19.1 & $0.5 ; 41.2$ & 0.044 \\
Low-grade inflammation & & & \\
$\Delta$ CRP & 0.8 & $-16.7 ; 18.2$ & 0.929 \\
$\Delta$ TNF- $\alpha$ & 16.4 & $-1.0 ; 36.8$ & 0.065 \\
$\Delta$ IL6 & -5.3 & $-19.4 ; 11.2$ & 0.501 \\
$\Delta$ Fibrinogen & -0.03 & $-0.19 ; 0.14$ & 0.751 \\
\hline
\end{tabular}

$\mathrm{Cl}$, confidence interval.

period and treatment sequence. We have analyzed the between-treatment effects as changes from first-period baseline. We choose this because the second-period baseline could be biased between the treatment sequences by possible differential carryover effects arising from the two treatments being stopped only 1 month earlier when completing the first-treatment period. In contrast, we did not have a specific reason to suspect such potential differential effects from previous glucose-lowering treatments at the first-period baseline (because previous such therapies were equally distributed between the treatment sequences (Table 1); $P>0.1$ ). However, additionally using the second-period baseline in the analyses did not substantially influence our results (data not shown). Finally, this trial was carried out with nonobese patients with type 2 diabetes. Therefore, the extent to which our findings may be extrapolated to obese patients remains uncertain. Of note is that more than $20 \%$ of Caucasian patients with type 2 diabetes are nonobese (32). In these patients and in contrast to those who are obese, solid scientific evidence supporting metformin as 'drug of first choice', as opposed to an insulin secretagogue, is however still lacking.

In conclusion, the improved glycemic control in type 2 diabetes induced by metformin and repaglinide is associated with a reduction in 3DG levels, which is similar in magnitude for both drugs and associated with reductions in AGEs and endothelial markers. This may constitute a metabolic pathway through which both treatments have a beneficial impact on the cardiovascular risk profile.

\section{Declaration of interest}

S S Lund, L Tarnow, H-H Parving, and A Vaag have reported equity in Novo Nordisk A/S. L Tarnow, O Pedersen, H-H Parving, and A Vaag have received funds from Novo Nordisk A/S for the research. S S Lund and A Vaag have received fees from Novo Nordisk A/S for speaking and A Vaag has received fees from Novo Nordisk A/S for organizing education. S S Lund, L Tarnow, H-H Parving, O Pedersen, and A Vaag are present or former employees of Steno Diabetes Center, Gentofte, Denmark. Steno Diabetes Center is an independent academic institution owned by Novo Nordisk A/S and The Novo Nordisk Foundation. The sponsors had no role in the study design, collection, analysis, or interpretation of data, writing the report, or the decision to submit the paper for publication.

\section{Funding}

Novo Nordisk A/S (Bagsværd, Denmark) and the Clinical Development Foundation at Steno Diabetes Center co-sponsored the study financially. Novo Nordisk A/S supplied repaglinide and repaglinide-placebo tablets. Hexal A/S (GEA Ltd) supplied metformin and metformin-placebo tablets. Dr I Ferreira was supported by a research grant from the Netherlands Heart Foundation (NHS; grant \# 2006T050).

\section{Acknowledgements}

We would like to thank the following people for great help and support in carrying out this study: Bente Blaaholm Nielsen as trial nurse; Merete Frandsen, Ulla Meng Smidt, Birgitte Vilsbøl Hansen, Tina Ragnholm Juhl, Berit Ruud Jensen, Lotte Pietraszek, Ingelise Rossing, and Jean Scheijen as laboratory technicians.

\section{References}

1 Brownlee M. Biochemistry and molecular cell biology of diabetic complications. Nature $2001 \quad \mathbf{4 1 4} 813-820$. (doi:10.1038/ 414813a)

2 Basta G. Receptor for advanced glycation endproducts and atherosclerosis: from basic mechanisms to clinical implications. Atherosclerosis 2008196 9-21. (doi:10.1016/j.atherosclerosis. 2007.07.025)

3 Yamagishi S, Nakamura K, Matsui T, Ueda S, Noda Y \& Imaizumi T. Inhibitors of advanced glycation endproducts (AGEs): potential utility for the treatment of cardiovascular disease. Cardiovascular Therapeutics 200826 50-58. (doi:10. 1111/j.1527-3466.2007.00038.x)

4 Thornalley PJ, Langborg A \& Minhas HS. Formation of glyoxal, methylglyoxal and 3-deoxyglucosone in the glycation of proteins by glucose. Biochemical Journal 1999344 109-116. (doi:10. 1042/0264-6021:3440109)

5 Kusunoki H, Miyata S, Ohara T, Liu BF, Uriuhara A, Kojima H, Suzuki K, Miyazaki H, Yamashita Y, Inaba K \& Kasuga M. Relation between serum 3-deoxyglucosone and development of diabetic microangiopathy. Diabetes Care 200326 1889-1894. (doi:10. 2337/diacare.26.6.1889)

6 Schindhelm RK, Alssema M, Scheffer PG, Diamant M, Dekker JM, Barto R, Nijpels G, Kostense PJ, Heine RJ, Schalkwijk CG \& Teerlink T. Fasting and postprandial glycoxidative and lipoxidative stress are increased in women with type 2 diabetes. Diabetes Care 200730 1789-1794. (doi:10.2337/dc06-2585)

7 Beisswenger PJ, Drummond KS, Nelson RG, Howell SK, Szwergold BS \& Mauer M. Susceptibility to diabetic nephropathy is related to dicarbonyl and oxidative stress. Diabetes $2005 \mathbf{5 4}$ 3274-3281. (doi:10.2337/diabetes.54.11.3274)

8 Thornalley PJ. Use of aminoguanidine (Pimagedine) to prevent the formation of advanced glycation endproducts. Archives of Biochemistry and Biophysics 2003419 31-40. (doi:10.1016/ j.abb.2003.08.013) 
9 Beisswenger PJ, Howell SK, Touchette AD, Lal S \& Szwergold BS. Metformin reduces systemic methylglyoxal levels in type 2 diabetes. Diabetes 199948 198-202. (doi:10.2337/diabetes.48.1.198)

10 Ruggiero-Lopez D, Lecomte M, Moinet G, Patereau G, Lagarde M \& Wiernsperger N. Reaction of metformin with dicarbonyl compounds. Possible implication in the inhibition of advanced glycation endproduct formation. Biochemical Pharmacology 1999 58 1765-1773. (doi:10.1016/S0006-2952(99)00263-4)

11 Rabbani N, Chittari MV, Bodmer CW, Zehnder D, Ceriello A \& Thornalley PJ. Increased glycation and oxidative damage to apolipoprotein B100 of LDL cholesterol in patients with type 2 diabetes and effect of metformin. Diabetes $2010 \mathbf{5 9} 1038-1045$. (doi:10.2337/db09-1455)

12 UK Prospective Diabetes Study (UKPDS) Group. Effect of intensive blood-glucose control with metformin on complications in overweight patients with type 2 diabetes (UKPDS 34). UK Prospective Diabetes Study (UKPDS) Group. Lancet 1998352 854-865. (doi:10.1016/S0140-6736(98)07037-8)

13 Lund SS, Tarnow L, Frandsen M, Smidt UM, Pedersen O, Parving $\mathrm{HH} \&$ Vaag AA. Impact of metformin versus the prandial insulin secretagogue, repaglinide, on fasting and postprandial glucose and lipid responses in non-obese patients with type 2 diabetes. European Journal of Endocrinology 2008158 35-46. (doi:10.1530/EJE-07-0500)

14 Lund SS, Tarnow L, Stehouwer CD, Schalkwijk CG, Frandsen M, Smidt UM, Pedersen O, Parving HH \& Vaag A. Targeting hyperglycaemia with either metformin or repaglinide in nonobese patients with type 2 diabetes: results from a randomized crossover trial. Diabetes, Obesity and Metabolism 20079 394-407. (doi:10.1111/j.1463-1326.2007.00713.x)

15 Lund SS, Tarnow L, Stehouwer CD, Schalkwijk CG, Teerlink T, Gram J, Winther K, Frandsen M, Smidt UM, Pedersen O, Parving HH \& Vaag AA. Impact of metformin versus repaglinide on non-glycaemic cardiovascular risk markers related to inflammation and endothelial dysfunction in non-obese patients with type 2 diabetes. European Journal of Endocrinology $2008 \mathbf{1 5 8}$ 631-641. (doi:10.1530/EJE-07-0815)

16 Schalkwijk CG, Posthuma N, ten Brink HJ, ter Wee PM \& Teerlink T. Induction of 1,2-dicarbonyl compounds, intermediates in the formation of advanced glycation end-products, during heatsterilization of glucose-based peritoneal dialysis fluids. Peritoneal Dialysis International $199919325-333$.

17 Schalkwijk CG, Ligtvoet N, Twaalfhoven H, Jager A, Blaauwgeers HG, Schlingemann RO, Tarnow L, Parving HH, Stehouwer CD \& van Hinsbergh VW. Amadori albumin in type 1 diabetic patients: correlation with markers of endothelial function, association with diabetic nephropathy, and localization in retinal capillaries. Diabetes 199948 2446-2453. (doi:10.2337/diabetes. 48.12.2446)

18 Teerlink T, Barto R, Ten Brink HJ \& Schalkwijk CG. Measurement of $N^{\varepsilon}$-(carboxymethyl)lysine and $N^{\varepsilon}$-(carboxyethyl)lysine in human plasma protein by stable-isotope-dilution tandem mass spectrometry. Clinical Chemistry 200450 1222-1228. (doi:10. 1373/clinchem.2004.031286)

19 De Jager J, Kooy A, Lehert P, Bets D, Wulffele MG, Teerlink T, Scheffer PG, Schalkwijk CG, Donker AJ \& Stehouwer CD. Effects of short-term treatment with metformin on markers of endothelial function and inflammatory activity in type 2 diabetes mellitus: a randomized, placebo-controlled trial. Journal of Internal Medicine 2005257 100-109. (doi:10.1111/j.1365-2796.2004. 01420.x)

20 Esposito K, Giugliano D, Nappo F \& Marfella R. Regression of carotid atherosclerosis by control of postprandial hyperglycemia in type 2 diabetes mellitus. Circulation 2004 110 214-219. (doi:10. 1161/01.CIR.0000134501.57864.66)
21 Manzella D, Grella R, Abbatecola AM \& Paolisso G. Repaglinide administration improves brachial reactivity in type 2 diabetic patients. Diabetes Care 200528 366-371. (doi:10.2337/diacare. 28.2.366)

22 Beisswenger PJ, Howell SK, O’Dell RM, Wood ME, Touchette AD \& Szwergold BS. $\alpha$-Dicarbonyls increase in the postprandial period and reflect the degree of hyperglycemia. Diabetes Care 200124 726-732. (doi:10.2337/diacare.24.4.726)

23 Ceriello A, Hanefeld M, Leiter L, Monnier L, Moses A, Owens D, Tajima N \& Tuomilehto J. Postprandial glucose regulation and diabetic complications. Archives of Internal Medicine 2004164 2090-2095. (doi:10.1001/archinte.164.19.2090)

24 Monnier L, Mas E, Ginet C, Michel F, Villon L, Cristol JP \& Colette C. Activation of oxidative stress by acute glucose fluctuations compared with sustained chronic hyperglycemia in patients with type 2 diabetes. Journal of the American Medical Association 2006 295 1681-1687. (doi:10.1001/jama.295.14.1681)

25 Niwa T. 3-Deoxyglucosone: metabolism, analysis, biological activity, and clinical implication. Journal of Chromatography. B, Biomedical Sciences and Applications 1999731 23-36. (doi:10. 1016/S0378-4347(99)00113-9)

26 Jono T, Nagai R, Lin X, Ahmed N, Thornalley PJ, Takeya M \& Horiuchi S. $N^{\varepsilon}$-(carboxymethyl)lysine and 3-DG-imidazolone are major AGE structures in protein modification by 3-deoxyglucosone. Journal of Biochemistry 2004136 351-358. (doi:10.1093/jb/ mvh124)

27 Schram MT, Chaturvedi N, Schalkwijk C, Giorgino F, Ebeling P, Fuller JH \& Stehouwer CD. Vascular risk factors and markers of endothelial function as determinants of inflammatory markers in type 1 diabetes: the EURODIAB Prospective Complications Study. Diabetes Care 200326 2165-2173. (doi:10.2337/diacare.26.7. 2165)

28 Schram MT, Chaturvedi N, Schalkwijk CG, Fuller JH \& Stehouwer CD. Markers of inflammation are cross-sectionally associated with microvascular complications and cardiovascular disease in type 1 diabetes - the EURODIAB Prospective Complications Study. Diabetologia 200548 370-378. (doi:10. 1007/s00125-004-1628-8)

29 de Jager J, Dekker JM, Kooy A, Kostense PJ, Nijpels G, Heine RJ, Bouter LM \& Stehouwer CD. Endothelial dysfunction and lowgrade inflammation explain much of the excess cardiovascular mortality in individuals with type 2 diabetes: the Hoorn Study. Arteriosclerosis, Thrombosis, and Vascular Biology $2006 \mathbf{2 6}$ 1086-1093. (doi:10.1161/01.ATV.0000215951.36219.a4)

30 Brouwers O, Niessen PM, Haenen G, Miyata T, Brownlee M, Stehouwer CD, De Mey JG \& Schalkwijk CG. Hyperglycaemiainduced impairment of endothelium-dependent vasorelaxation in rat mesenteric arteries is mediated by intracellular methylglyoxal levels in a pathway dependent on oxidative stress. Diabetologia 201053 989-1000. (doi:10.1007/s00125-010-1677-0)

31 Stirban A, Negrean M, Stratmann B, Gawlowski T, Horstmann T, Gotting C, Kleesiek K, Mueller-Roesel M, Koschinsky T, Uribarri J, Vlassara H \& Tschoepe D. Benfotiamine prevents macro- and microvascular endothelial dysfunction and oxidative stress following a meal rich in advanced glycation endproducts in individuals with type 2 diabetes. Diabetes Care 200629 2064-2071. (doi:10.2337/dc06-0531)

32 Dalton M, Cameron AJ, Zimmet PZ, Shaw JE, Jolley D, Dunstan DW \& Welborn TA. Waist circumference, waist-hip ratio and body mass index and their correlation with cardiovascular disease risk factors in Australian adults. Journal of Internal Medicine 2003254 555-563. (doi:10.1111/j.1365-2796.2003.01229.x)

Received 6 December 2010

Accepted 4 January 2011 